\title{
Synthetic Methanol and Formate Assimilation Via Modular Engineering and Selection Strategies
}

\author{
Nico J. Claassens, Hai He and Arren Bar-Even*
}

\author{
Max Planck Institute of Molecular Plant Physiology, Potsdam-Golm, Germany. \\ ${ }^{*}$ Correspondence: bar-even@mpimp-golm.mpg.de \\ https://doi.org/10.21775/cimb.033.237
}

\begin{abstract}
One-carbon (C1) feedstocks can provide a vital link between cheap and sustainable abiotic resources and microbial bioproduction. Soluble $\mathrm{C} 1$ substrates - methanol and formate - could prove to be more suitable than gaseous feedstocks as they avoid mass transfer barriers. However, microorganisms that naturally assimilate methanol and formate are limited by a narrow product spectrum and a restricted genetic toolbox. Engineering biotechnological organisms to assimilate these soluble $\mathrm{C} 1$ substrates has therefore become an attractive goal. Here, we discuss the use of a step-wise, modular engineering approach for the implementation of $\mathrm{C} 1$ assimilation pathways. In this strategy, pathways are divided into metabolic modules, the activities of which are selected for in dedicated gene-deletion strains whose growth directly depends on module activity. This provides an easy way to identify and resolve metabolic barriers hampering pathway performance. Optimization of gene expression levels and adaptive laboratory evolution can be used to establish the desired activity if direct selection fails. We exemplify this approach using several pathways, focusing especially on the ribulose monophosphate cycle for methanol assimilation and the reductive glycine pathway for formate assimilation. We argue that such modular engineering and selection strategies will prove essential for rewiring microbial metabolism towards new growth phenotypes and sustainable bioproduction.
\end{abstract}

\section{Introduction}

One-carbon (C1) compounds could prove to be a crucial link between the abiotic and the biotic worlds. These feedstocks can be obtained from low-cost and abundant sources, such as syngas and natural gas (Dürre and Eikmanns, 2015; Clomburg et al., 2017), and can be produced directly from $\mathrm{CO}_{2}$ using energy sources such as sunlight and renewable electricity (Kumar et al., 2012; Martín et al., 2015; Claassens et al., 2018; Jouny et al., 2018). Multiple microorganisms can be cultivated on C1 compounds as sole carbon and energy sources, thus opening new avenues for sustainable bioproduction.

However, the use of microorganisms that can naturally grow on $\mathrm{C} 1$ substrates is limited by multiple factors, including a narrow product spectrum, low yields, titres, and productivities, a restricted genetic toolbox for engineering, and gaps in our understanding of their cellular physiology and metabolism (Whitaker et al., 2015; Clomburg et al., 2017). To overcome these difficulties, recent metabolic engineering efforts are aiming to introduce C1 assimilation pathways into model biotechnological microorganisms that are easier to engineer and that can be better optimized for industrially relevant conditions. These efforts use either natural pathways that are known to sustain high yields, or, more boldly, synthetic pathways with low ATP cost that could theoretically support increased yields (Bar-Even et al., 2013; Siegel et al., 2015; Bar-Even, 
2016;). Some of these synthetic pathways can be established by combining naturally existing enzymes, while others include novel enzyme activities that can be realized by protein engineering (Erb et al., 2017). In fact, engineered enzymes have already been demonstrated in vitro to support formate assimilation (Siegel et al., 2015) and carbon fixation (Schwander et al., 2016).

In this review, we discuss metabolic engineering studies aiming to introduce pathways for the assimilation of the soluble $\mathrm{C} 1$ compounds methanol and formate, the utilization of which bypasses the challenges associated with mass transfer of gaseous $\mathrm{C} 1$ substrates, such as methane and carbon monoxide (Henstra et al., 2007; Fei et al., 2014). We specifically focus on modular and selectionbased engineering strategies in which the activity of pathway segments is coupled to cellular growth. We show that this step-wise approach is vital for the realization of synthetic $\mathrm{C} 1$ assimilation.

\section{Modularity and selection as metabolic engineering strategies}

Engineering synthetic $\mathrm{C} 1$ metabolism requires the overexpression of pathway enzymes, especially those that are missing in the host or that are natively expressed at insufficient levels. However, simple overexpression is unlikely to be sufficient for realizing the activity of the entire pathway. This is mainly because of the overlap between the introduced pathway and the host central metabolism, resulting in disrupted fluxes through both systems. To better identify and resolve problematic metabolic interactions, it is helpful to divide the synthetic pathway into smaller metabolic modules, i.e. subpathways consisting of several reactions (Fig. 14.1). The in vivo implementation of these modules can be considerably easier than the full pathway and provide vital information on the metabolic context that enables or constrains the newly introduced

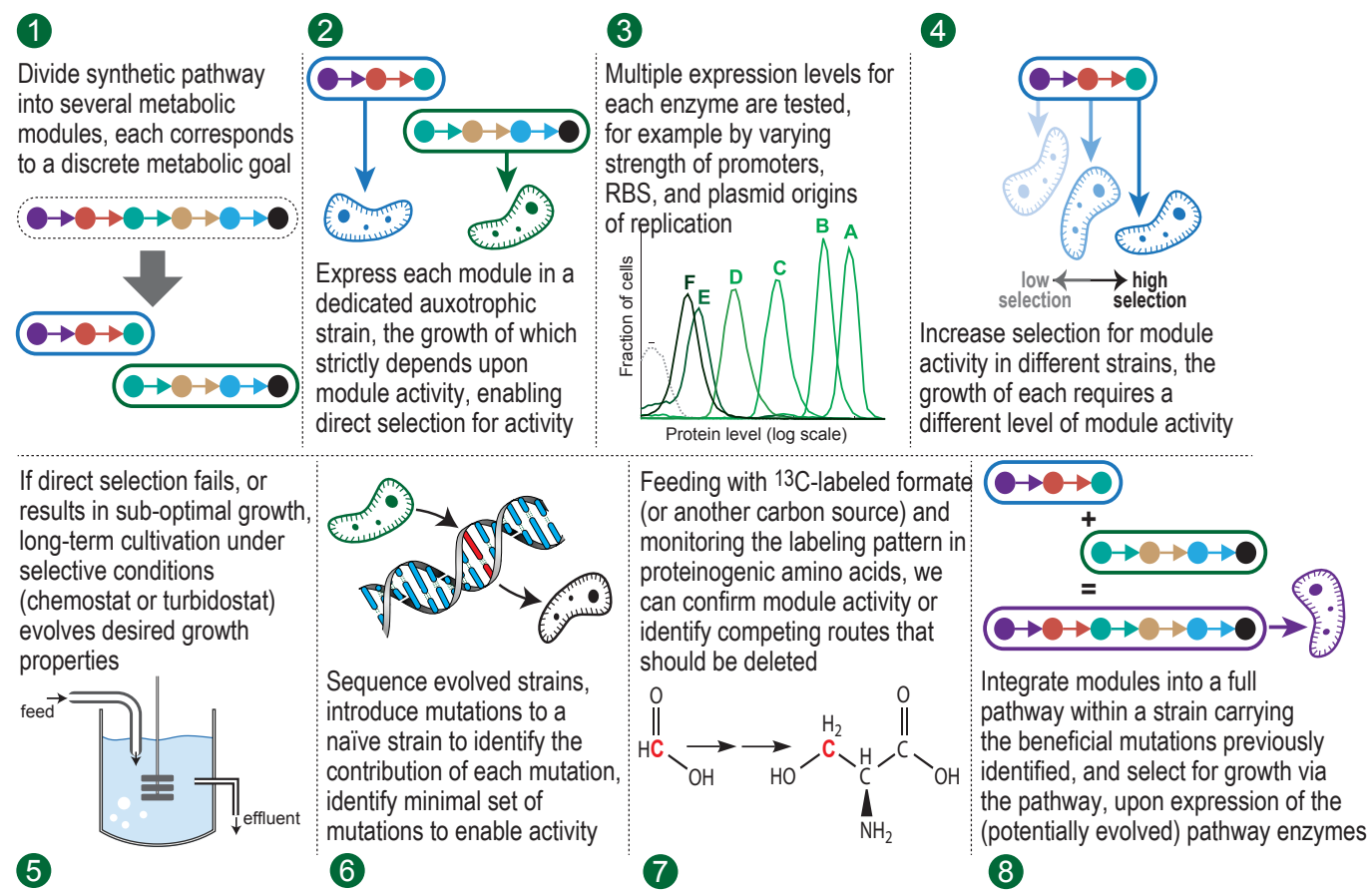

Figure 14.1 A schematic representation of the modular engineering and selection approach outlined in this paper. 
activities. To probe the implementation of metabolic modules, it is useful to couple their activity with the growth of the host.

Coupling module activity with growth usually requires modifying the metabolic network of the host by performing strategic gene deletions. These are made to generate a strain auxotrophic for certain essential metabolic intermediates - for example, an amino acid - which can be exclusively synthesized via the synthetic module. As a result, cellular growth becomes dependent on the activity of the module. A range of selection strains can be designed with increasing selection pressure for pathway activity: a 'minimal' selection is sustained if the module provides a single required metabolite, higher selection pressure is obtained when module activity is responsible for the biosynthesis of multiple building blocks, and very high selection pressure is imposed when the biosynthesis of all or most biomass is dependent on the module.

The design of modules and selection strains can be assisted by computational tools based on Flux Balance Analysis, for example OptKnock or FlexFlux (Burgard et al., 2003; Marmiesse et al., 2015; Meyer et al., 2018). Yet, in most cases, manual design based on biochemical and metabolic knowledge suffices. Specifically, when dividing a pathway into metabolic modules, several factors should be taken into consideration (Wenk et al., 2018): (1) the module should be linked to a clear growth phenotype within an appropriate selection strain, resulting in growth versus no growth readout; (2) the number of enzymes in a module should be limited, to enable easy expression and optimization, and to allow straightforward interpretation of growth phenotypes; (3) modules should together cover the whole pathway and could overlap with one another, such that enzymes occurring in multiple modules can be tested in different metabolic contexts; (4) ideally, modules should be easy to combine, that is, dedicated selection strains - whose growth is dependent on the activity of several consecutive modules - should be easy to construct.

When direct selection for module activity fails or results in poor growth, further optimization is required (Fig. 14.1). This can be achieved by modulating the expression levels of the pathway enzymes (Zelcbuch et al., 2013; Wenk et al., 2018), and potentially also of related host enzymes, e.g. deletion or down-regulation of enzymes that divert metabolic intermediates from the pathway. In addition, different enzyme variants or codon optimization of the relevant genes can support increased expression and activity. Another method, which does not rely on genetic tools, is the addition of small molecules that specifically inhibit interfering enzymes, as demonstrated for the glycolytic glyceraldehyde 3-phosphate dehydrogenase in the engineering of methanol assimilation in Escherichia coli (Woolston et al., 2018a).

If these approaches fail to establish module activity, adaptive laboratory evolution (ALE) can be performed to increase module functionality and establish module-dependent growth (Portnoy et al., 2011). For this process, the overexpressed genes should preferably be integrated into the genome rather than carried on a plasmid as to increase the chance of beneficial mutations to be fixed in the population. Different types of ALE can be applied; a prominent approach being continuous cultivation on a selective medium, with limiting amounts of the compounds for which the cells are auxotrophic. This method was applied for the successful engineering of the $\mathrm{CO}_{2}$-fixing Calvin cycle in E. coli (Antonovsky et al., 2016), where the concentration of the limiting substrate xylose was gradually decreased as growth improved. Another approach involves swapping between permissive and selection media, where the former contains the auxotrophy-related compounds and the latter does not (Marlière et al., 2011). The relative dosages of the different media are coupled to the growth of the population, such that increased cell density leads to addition of selection medium, and a decrease in cell density results in more permissive medium. Such cultivation regime adapts the population towards using the selection medium until the permissive medium is no longer required (Marlière et al., 2011; Bouzon et al., 2017; Döring et al., 2018). Following successful evolution of module activity, the evolved genomes can be sequenced to identify the accumulated mutations. The specific effect of different mutations can be interpreted and further studied by reintroducing them into a non-evolved selection strain. 


\section{Synthetic methanol assimilation}

Methanol can be produced from diverse fossil and renewable sources and has been proposed as a promising feedstock for industrial applications and microbial growth (Olah et al., 2009; Olah, 2013; Zhang et al., 2017). Hence, biological conversion of methanol to products has received considerable attention (Schrader et al., 2009; Whitaker et al., 2015; Pfeifenschneider et al., 2017; Bennett et al., 2018a). As genetic toolboxes for the engineering of most promising natural methylotrophs (e.g. Bacillus methanolicus) are still underdeveloped, engineering model biotechnological microbes for growth on methanol has become an attractive target.

Three native pathways are known to support growth on methanol: the ribulose monophosphate (RuMP) cycle, the xylulose monophosphate cycle, and the serine pathway (Kato et al., 2006; Chistoserdova et al., 2009). Among these, the engineering of the RuMP cycle has received most attention, as this route supports the highest yield (Bar-Even et al., 2013). The heterologous establishment of the serine pathway has not yet been reported and only a single study aimed at engineering the xylulose monophosphate pathway in Saccharomyces cerevisiae (Dai et al., 2017).

A synthetic methanol assimilation pathway has also been proposed, where three formaldehyde molecules are condensed to dihydroxyacetone by a rationally engineered formolase enzyme. This pathway was recently introduced in E. coli, but did not lead to substantial methanol assimilation, probably due to the poor kinetics of the formolase enzyme (Wang et al., 2017). Another proposed synthetic methanol condensation cycle, which was demonstrated in vitro, consists of the RuMP cycle combined with non-oxidative glycolysis (Bogorad et al., 2014). In this pathway, fructose-6-phosphate (F6P) is cleaved by phosphoketolases to produce acetyl-CoA, bypassing pyruvate decarboxylation and preventing carbon loss. Finally, an alternative, synthetic structure of the serine pathway was proposed, in which serine is deaminated to pyruvate, and glycine is recycled via threonine biosynthesis and degradation, further generating acetyl-CoA as a biomass precursor (Bar-Even, 2016). A recent study describes the successful selection for key metabolic modules of this synthetic route, that is, formate assimilation into serine via the tetrahydrofolate (THF) system and glycine production solely from threonine cleavage (Yishai et al., 2017).

As the RuMP cycle (Fig. 14.2a) attracts the most research attention so far, we will focus on attempts for its engineering in biotechnological hosts. Non-methylotrophic hosts typically lack only three enzymes of the pathway: methanol dehydrogenase, hexulose-6-phosphate synthase and 6-phospho-3-hexulosiomerase. Several studies have overexpressed these enzymes in $E$. coli (Müller et al., 2015; Price et al., 2016; Whitaker et al., 2017; Bennett et al., 2018b; Gonzalez et al., 2018) and Corynebacterium glutamicum (Leßmeier et al., 2015; Witthoff et al., 2015) and have demonstrated methanol assimilation, albeit at low rates and assimilation efficiencies. These efforts, however, did not apply a selection strategy, that is, cellular growth was not dependent on methanol assimilation.

The RuMP cycle can be divided into three main modules: methanol oxidation to formaldehyde, formaldehyde assimilation into central metabolism, and regeneration of the acceptor metabolite ribulose 5-phosphate (Ru5P) (Fig. 14.2b). In the assimilation module, formaldehyde is condensed with $\mathrm{Ru} 5 \mathrm{P}$ to generate F6P, which can be metabolized to all biomass building blocks. The assimilation and regeneration modules can be supported by several alternative metabolic structures, the most efficient one uses glycolysis and the non-oxidative pentose phosphate pathway (Quayle and Ferenci, 1978; Zhang et al., 2017).

Several recent studies have attempted direct selection for the activities of the methanol oxidation module and the formaldehyde assimilation module in E. coli (Chen et al., 2018; Meyer et al., 2018) and C. glutamicum (Tuyishime et al., 2018). These studies generated a selection strain in which ribose 5-phosphate isomerase is deleted $(\triangle r p i A B)$. This knockout blocked growth on xylose or gluconate (the latter with the additional deletion of $e d d$, encoding phosphogluconate dehydratase). The methanol oxidation and formaldehyde assimilation modules were expected to rescue growth on xylose or gluconate by enabling their assimilation via a 'RuMP shunt', a linear route converting Ru5P to F6P via condensation with formaldehyde (Fig. $14.2 \mathrm{c}$ and $\mathrm{d}$ ). Yet, none of these studies was able to demonstrate the required activity upon direct 
(a)

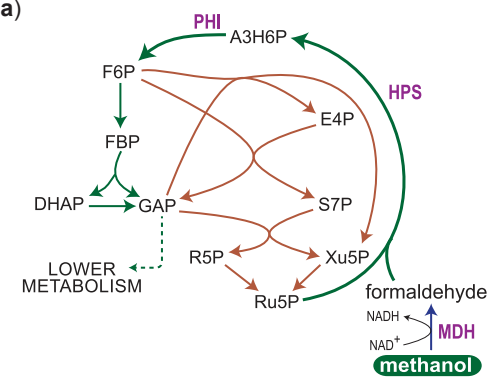

(c)

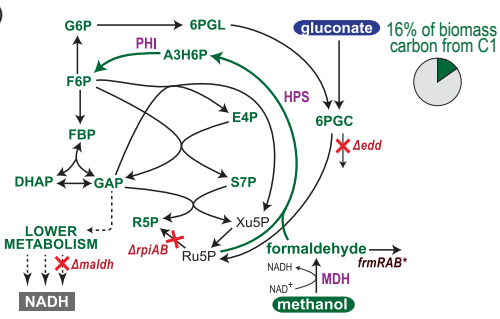

(e)

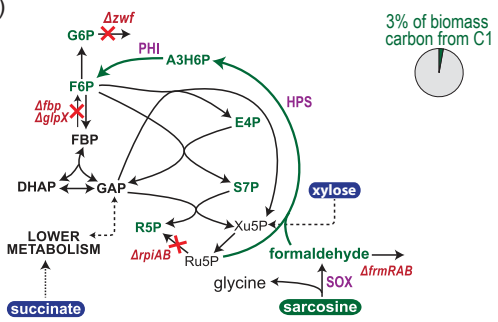

(g)

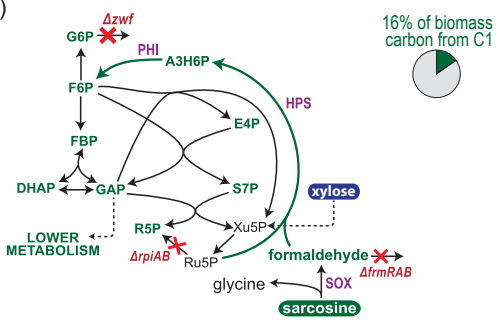

(b)

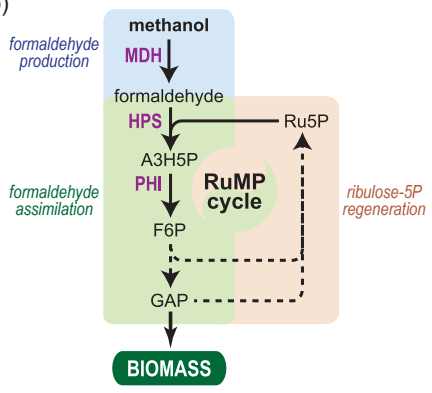

(d)

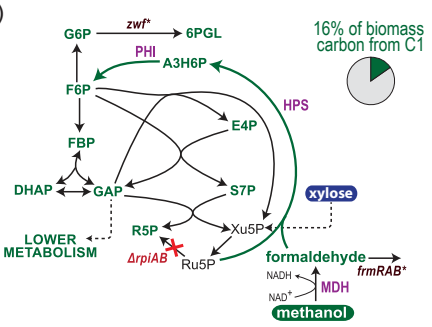

(f)

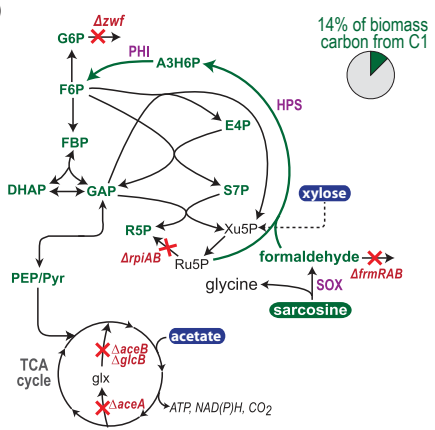

(h)

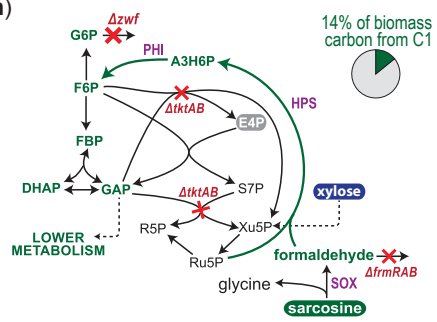

Figure 14.2 Engineering the RuMP shunt in E. coli. (a) Metabolic structure of a variant of the RuMP cycle. (b) Subdivision of the RuMP cycle into metabolic modules; (c, d) Selection schemes for methanol-dependent growth of E. coli via the RuMP shunt as described by Meyer et al. (2018) (c) and Chen et al. (2018) (d). (e-h) Selection schemes for growth via the RuMP shunt at different selection strengths as described in He et al. (2018). Pie charts indicate the minimum fraction of carbons in biomass that are derived from $\mathrm{C} 1$ (formaldehyde or methanol) in the different selection schemes, as calculated using (Neidhardt et al., 1990). Gene deletions are shown in red; overexpressed enzymes in purple; enzymes that mutated during ALE in brown; metabolites that are dependent on the RuMP shunt in green; and substrates that have to be co-fed in blue. Enzyme abbreviations: aceA, isocitrate lyase; aceB, malate synthase $B$; edd, phosphogluconate dehydratase; fbp; fructose-1,6-bisphosphatase; frmRAB, glutathione-dependent formaldehyde detoxification system; glcB, malate synthase G; glpX, fructose-1,6-bisphosphatase 2; HPS, 3-hexulose-6-phosphate synthase; maldh, malate dehydrogenase; $\mathrm{MDH}$, methanol dehydrogenase; $\mathrm{PHI}, 6$-phospho-3-hexuloisomerase; rpiAB, ribose 5-phosphate isomerase; SOX, sarcosine oxidase; tktAB, transketolase A and B; zwf, glucose 6-phosphate dehydrogenase. Metabolite abbreviations: A3H6P, arabino 3-hexulose 6-phosphate; DHAP, dihydroxyacetone phosphate; E4P, erythrose 4-phosphate; F6P, fructose 6-phosphate; FBP, fructose bisphosphate; GAP, glyceraldehyde 3-phosphate; 6PGC, 6-phospho-gluconate; 6PGL, 6-phospho-glucono-lactone; R5P, ribose 5-phosphate; Ru5P, ribulose 5-phosphate; S7P, sedoheptulose 7-phosphate; Xu5P, xylulose 5-phosphate. 
selection, and ALE was necessary to establish methanol-assimilation-dependent growth.

The strict requirement for ALE to achieve RuMP shunt-dependent growth can be attributed to the unfavorability of methanol oxidation. Supporting this notion, all of these studies found mutations linked to a decreased $\mathrm{NADH} / \mathrm{NAD}$ ratio, e.g. by interrupting or inhibiting the TCA cycle (Chen et al., 2018; Meyer et al., 2018; Tuyishime et al., 2018). This likely relates to the fact that methanol oxidation is thermodynamically limited by a high $\mathrm{NADH} / \mathrm{NAD}$ ratio. Using deuterated methanol $\left(\mathrm{CD}_{3} \mathrm{OD}\right)$, it was shown that methanol dehydrogenase also kinetically limits the activity of the RuMP shunt in E. coli (Woolston et al., 2018a). Methanol oxidation could be improved by identifying or engineering kinetically superior variants, which could be directly screened using a formaldehyde biosensor (Woolston et al., 2018b) or selected for in appropriate selection strains.

To overcome the barriers associated with methanol oxidation, we decided to separate the formaldehyde production module from that of formaldehyde assimilation, such that we could test the latter in more detail. Towards this aim, we replaced methanol dehydrogenase with the kinetically efficient and thermodynamically favourable sarcosine oxidase that metabolizes sarcosine to formaldehyde and glycine (He et al., 2018). We further constructed several selection strains, imposing different levels of selective pressure on formaldehyde assimilation via the RuMP shunt. These included (i) $\triangle f r m R A B \quad \Delta r p i A B \quad \Delta f b p \Delta g l p X \Delta z w f$ strain in which the synthetic shunt provides only essential sugar phosphates while succinate serves as the main growth substrate (Fig. 14.2e); (ii) $\triangle$ frmRAB $\triangle r p i A B$ $\triangle a c e B A \Delta g l c B \Delta z w f$ strain in which the RuMP shunt provides almost all cellular building blocks while acetate oxidation provides reducing power and energy (Fig. 14.2f); (iii) $\triangle$ frmRAB $\Delta r p i A B \Delta z w f$ strain, where the shunt is responsible for satisfying all cellular carbon and energy needs (Fig. 14.2g); and (iv) $\triangle$ frmRAB $\triangle t k t A B \Delta z w f$ strain in which almost all cellular carbon and energy needs are supported by the RuMP shunt (Fig. 14.2h).

We were able to directly select for growth of all of these strains via the RuMP shunt without the need for ALE (He et al., 2018), most probably because our selection scheme bypasses the challenge of methanol oxidation. However, the growth we obtained with the $\triangle f r m R A B \triangle r p i A B \Delta z w f$ strain was considerably poorer than that observed using the $\triangle$ frmRAB $\Delta t k t A B \Delta z w f$ strain. This serves as a clear demonstration of the importance of the metabolic context for establishing activity of a newly introduced pathway. In this specific case, the poor growth associated with the $\triangle$ frmRAB $\triangle$ rpiAB $\Delta z w f$ strain probably stems from costly metabolism of F6P 'back' to xylulose-5-phosphate and Ru5P, and potentially from the inhibition of essential transketolase reactions due to the accumulation of the coproduct xylulose-5-phosphate (He et al., 2018).

The major challenge of establishing a fully functional RuMP cycle lies in the regeneration module. To make things even more difficult, the establishment of autocatalytic cycles, such as the RuMP cycle, requires the kinetic parameters of enzymes to be carefully balanced as to avoid draining the pathway intermediates (Barenholz et al., 2017). A recent study suggested an interesting way to tackle this challenge, by overexpressing the irreversible SBPase to force flux towards the regeneration of Ru5P (Woolston et al., 2018a). Another approach is to overexpress the enzymes of the pentose phosphate pathway from an organism that supports more efficient regeneration of RuSP (Bennett et al., 2018b). Still, as was previously shown with ALE of E. coli to achieve a functional Calvin Cycle, down-regulation of branching enzymes, e.g. ribosephosphate diphosphokinase, might be necessary to establish a sustainable cyclic flux (Antonovsky et al., 2017; Herz et al., 2017).

\section{Synthetic formate assimilation}

Formate is a promising microbial feedstock that can be efficiently produced from $\mathrm{CO}_{2}$ by electrochemical and photochemical processes (Kopljar et al., 2016; Zhou et al., 2016; Yang et al., 2017). Formate can also be obtained from partial oxidation of biomass or natural gas and by hydrogenation of $\mathrm{CO}_{2}$ (Shen et al., 2015; Wang et al., 2015).

Microbial growth on formate as a carbon and energy source is reported for diverse groups of microorganisms. Acetogenic and methanogenic microbes can grow on formate using the reductive acetyl-CoA pathway (Kerby and Zeikus, 1987), representing the most efficient way to convert this feedstock into a product (Bar-Even et al., 2013). 
However, this anaerobic pathway can support only a narrow product spectrum (Bertsch and Müller, 2015). Aerobic growth on formate is sustained by the Calvin cycle and the serine pathway; however, the high ATP costs of these pathways reduce the potential biomass and product yields (Bar-Even et al., 2013). To overcome this problem, several synthetic pathways have been designed to support high yields under aerobic conditions (Bar-Even et al., 2013; Siegel et al., 2015; Bar-Even, 2016).

In the synthetic formolase pathway, formate is first reduced to formaldehyde by promiscuous activity of two enzymes: acetyl-CoA synthase and acetylaldehyde dehydrogenase (Siegel et al., 2015). Then, three formaldehyde molecules are condensed into dihydroxyacetone by the formolase enzyme described before. The activity of the pathway was demonstrated in vitro, but its in vivo functionality was very poor due to the low activities of formaldehyde dehydrogenase and the formolase enzyme (Siegel et al., 2015). To boost the activities of these limiting enzymes, a modular selection strategy can be used. For example, a module responsible for formate reduction to formaldehyde can be tested in a formaldehyde-dependent strain - for example, the RuMP shunt-dependent strains described above - and ALE can be used to increase this activity. Similarly, activity of the formolase enzyme might be initially tested and optimized in a strain which produces formaldehyde via sarcosine oxidation and assimilate dihydroxyacetone to provide only a fraction of cellular building blocks. For example, by deleting phosphoglycerate mutase, central metabolism can be divided into upper and lower segments, where succinate provides carbon and energy for lower metabolism and dihydroxyacetone phosphorylation provides carbon only for upper metabolism (Zelcbuch et al., 2015). This would impose moderate selection for the activity of the formolase enzyme and would thus be a more reasonable initial target.

A more feasible approach to establish formate assimilation would be to focus on existing enzymatic activities that could be combined to realize a new pathway. This is exactly the case of the synthetic reductive glycine pathway (Fig. 14.3a) (Bar-Even et al., 2013). All the reactions of this pathway are catalysed by known and ubiquitous enzymes. It is even possible that the pathway operates endogenously in some microbes (Figueroa et al., 2017). Its limited overlap with central metabolism and its very high ATP-efficiency further make the reductive glycine pathway especially promising to support aerobic formate assimilation.

To facilitate the implementation of the reductive glycine pathway in E. coli, we divided it into four modules (Fig. 14.3a): (i) a C1 activation module in which formate is condensed with the THF system and reduced to 5,10-methylene-THF; (ii) a glycine biosynthesis module that condenses the $\mathrm{C} 1$ moiety from 5,10-methylene-THF with $\mathrm{CO}_{2}$ and ammonia to generate the $\mathrm{C} 2$ metabolite glycine; (iii) a serine biosynthesis module that condenses glycine with another $\mathrm{C} 1$ moiety to give the $\mathrm{C} 3$ metabolite serine; and (iv) a serine assimilation module that deaminates this amino acid to produce pyruvate as a biomass precursor.

We constructed several selection strains to demonstrate the activities of the pathway modules in E. coli (Yishai et al., 2017, 2018). First, we constructed a C1-auxotroph strain $(\Delta g l y A \Delta g c v T H P)$ and showed that overexpression of formate THF ligase (FTL) enabled formate to serve as sole source of all C1-dependent building blocks, including purines, thymidine, and methionine (Fig. $14.3 \mathrm{~b})$. This confirmed the efficient activity of the C1-activation module. Next, we selected for the combined activity of the $\mathrm{C} 1$-activation module and the serine biosynthesis module. Towards this aim, we constructed a strain deleted in 3-phosphoglycerate dehydrogenase $(\Delta s e r A$, the first enzyme of serine biosynthesis) and the glycine cleavage system $(\Delta g c v T H P)$. Only upon overexpression of FTL and the native bifunctional 5,10-methenyl-THF cyclohydrolase/5,10-methylene-THF dehydrogenase (FolD), glycine and formate could serve as $\mathrm{C} 1$ and serine sources to support cell growth (Fig. 14.3c). This demonstrates that testing a module in this case the $\mathrm{C} 1$ activation module - in different metabolic contexts is important to uncover hidden bottlenecks: while the endogenous activity of FolD sufficed for the first selection strain, the higher activity required for the growth of the second selection strain necessitated dedicated overexpression of the enzyme (Yishai et al., 2017).

In a follow-up study, we selected for the activities of the C1-assimilation module and the glycine biosynthesis module (Yishai et al., 2018). We constructed a strain auxotrophic to $\mathrm{C} 1$ and glycine $(\Delta g l y A \Delta l t a E \Delta k b l \Delta a c e A)$. We could establish 
(b)

(a)

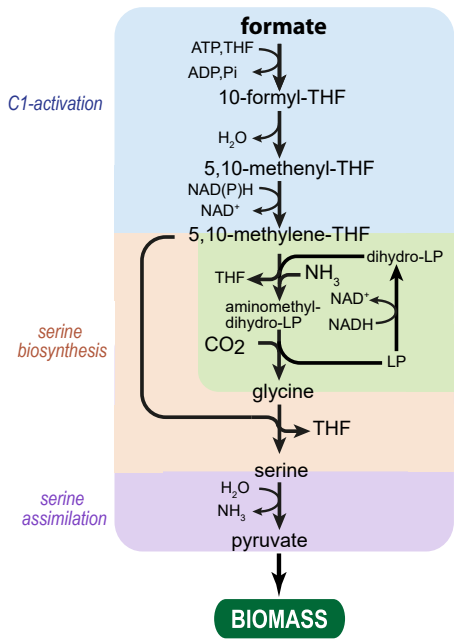

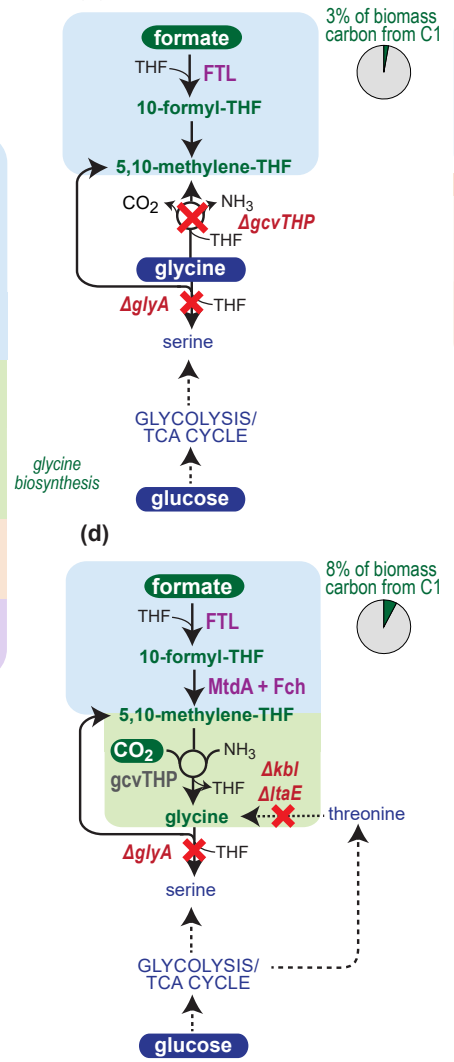

(c)

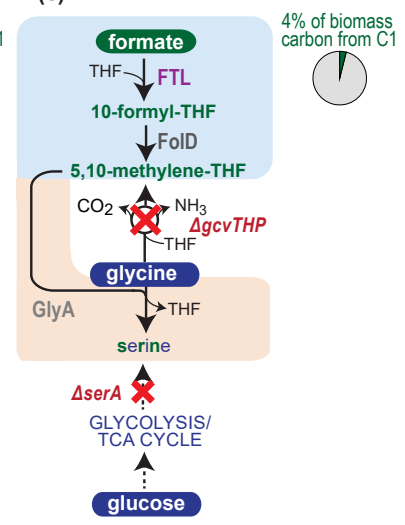

(e)

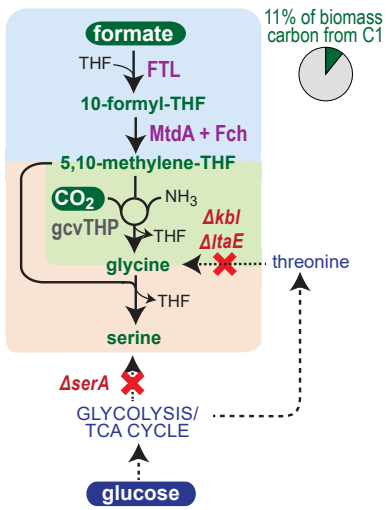

Figure 14.3 Engineering the reductive glycine pathway in E. coli. (a) Metabolic scheme of the reductive glycine pathway and its subdivision into modules; $(b-e)$ Selection schemes for the activity of different modules of the reductive glycine pathway, as demonstrated in E. coli (Yishai et al., 2017, 2018). Pie charts indicate the minimum fraction of carbons in biomass that are derived from formate and $\mathrm{CO}_{2}$ in the different selection schemes, as calculated using (Neidhardt et al., 1990). Gene deletions are shown in red; overexpressed foreign enzymes in purple; overexpressed native enzymes in grey; metabolites that are produced from formate and $\mathrm{CO}_{2}$ in green; and substrates that have to be co-fed in blue. Abbreviations: Fch, 5,10-methenyl-THF cyclohydrolase; FolD, bifunctional 5,10-methenyl-THF cyclohydrolase/5,10-methylene-THF dehydrogenase; FTL, formateTHF ligase; gcvTHP(L), glycine cleavage system subunits $\mathrm{T}, \mathrm{H}$ and $\mathrm{P}$ (and lipoamide dehydrogenase subunit); glyA, serine hydroxymethyltransferase; ItaE, threonine aldolase; kbl, 2-amino-3-ketobutyrate CoA ligase; LP, lipoamide-protein; MtdA, 5,10-methylene-THF dehydrogenase; serA, 3-phosphoglycerate dehydrogenase; THF, tetrahydrofolate.

formate conversion to C1-activated-THF and glycine upon overexpression of the enzymes of the native glycine cleavage system as well as FTL, 5,10-methenyl-THF cyclohydrolase, and 5,10-methylene-THF dehydrogenase from Methylobacterium extorquens (Fig. 14.3d). The latter two enzymes were necessary to replace the native FolD, whose activity was too low to support the required flux even when overexpressed, probably since it is inhibited by the key intermediate 10-formyl-THF (Yishai et al., 2018). Next, to select for the activity of three of the pathway modules, we constructed a C1-glycine-serine auxotrophic strain $(\Delta \operatorname{ser} A$ $\Delta l t a E \Delta k b l \Delta a c e A)$ (Fig. 14.3e). Overexpression of enzymes mentioned above resulted in $\mathrm{C} 1$, glycine and serine production solely from formate and $\mathrm{CO}_{2}$. Overall, $\approx 10 \%$ of carbons in biomass were provided by the pathway and the fast growth obtained (doubling rate of $\approx 1.7$ hours) indicates high activity of all pathway components.

Another recent study also focused on modular engineering of the reductive glycine pathway in $E$. coli (Tashiro et al., 2018). Similar to the approach described above, this study demonstrated that 
the overexpression of three foreign genes from Clostridium ljungdahlii can support $\mathrm{C} 1$ and serine biosynthesis from formate and glycine (Fig. 14.3c). However, for demonstration of glycine and serine biosynthesis via the reductive activity of the glycine cleavage system, leaky auxotrophic strains were employed in which only serA or $g l y A$ were deleted, while threonine cleavage to glycine (via $\mathrm{Kbl}$ or LtaE) was left untouched. Consequently, as is supported by the labelling data, in these strains, the majority of glycine and serine were most probably derived from threonine cleavage rather than formate assimilation.

In a parallel effort to the rational engineering approach, we demonstrated the establishment of the three modules of the reductive glycine pathway using ALE (Döring et al., 2018). This work used the same tight selection strategies as above but introduced only a single foreign enzyme: Clostridial FTL that was integrated into the genome. The cells were cultivated continuously and provided with two types of alternating media: a selection medium (which does not contain the auxotrophyrelieving compounds) that was added when the culture turbidity surpassed a threshold, and a permissive medium (containing the compounds for which the strains are auxotrophic) that was supplied upon decrease of the culture turbidity below this threshold. This procedure was used to select for growth on the stressing medium and, using sequential rounds of evolution, established metabolism of formate to C1-activated-THF, glycine, and, finally, serine. Genome sequencing of these strains revealed several mutations, including in the coding region of the key limiting enzyme FolD. Several genes were duplicated in the genome, including FTL and FolD, probably to increase their expression levels. This study confirms that the same metabolic goals can be achieved either via a rational engineering approach or via long term evolution.

To complete the establishment of the reductive glycine pathway, the next challenge is to integrate the first three modules - converting formate to serine - with the last one, that is, serine assimilation into central metabolism. This would require an order of magnitude increase in the flux via the pathway and would most probably require ALE to adapt the cellular physiology for the novel growth mode.

\section{Conclusions}

Recent efforts using modular and selection-based engineering approach have enabled C1-routes to support the biosynthesis of a substantial fraction of the host's biomass. These studies demonstrate a general approach of iterative design, build, test and learn cycles. First, pathway modules and selection strains are designed and constructed. Testing module activity using these selection strains provides insights into the metabolic constraints that limit flux, from which better designs and improved activity can emerge. Selection strains are therefore an essential tool for these cycles, as they provide simple performance readout that facilitates optimization of module and pathway activity.

As mentioned above for the RuMP cycle, a major challenge for establishing $\mathrm{C} 1$ pathways relates to the regulation of flux that is diverted away from the pathway towards other biosynthesis routes. This is especially true for autocatalytic cycles in which the product is also an intermediate of the pathway. To realize stable activity, proper balancing is required between the rates of pathway enzymes and those of the branching enzymes. As previously demonstrated, for autocatalytic cycles, this likely requires ensuring low affinities of the branching enzymes towards the pathway metabolites (Barenholz et al., 2017). Indeed, the use of ALE for the establishment of a functional Calvin cycle in E. coli resulted in lower activity of branching reactions (Antonovsky et al., 2016). Fortunately, branching reactions are easy to identify such that a rational design approach might be able to achieve the same goals as ALE, e.g. genetic or protein engineering aiming at lower expression levels or affinities of branching enzymes could directly establish the desired growth phenotype.

Most efforts to establish synthetic C1 metabolism have so far focused on the RuMP cycle, the reductive glycine pathway, and the formolase pathway. Yet, given the diversity of metabolic solutions for $\mathrm{C} 1$ assimilation, more pathways are likely to be designed and tested, and the modular selection approach described here would be vital for their implementation. Currently, E. coli serves as the prime host for engineering synthetic $\mathrm{C} 1$ assimilation, mainly due to the highly developed genetic toolbox for its engineering and our extensive knowledge of its physiology and metabolism. However, other microbial hosts might prove to be more 
suitable. Specifically, some microbes can produce specific chemicals better than E. coli and others display higher tolerance towards substrates or products. For example Corynebacterium glutamicum is arguably the best host to produce amino acids (Wendisch et al., 2006), and Saccharomyces cerevisiae can tolerate high concentrations of the formate feedstock and alcohol products (Overkamp et al., 2002; Mohd Azhar et al., 2017). Also, some metabolic modules can directly integrate with $\mathrm{C} 1$ metabolism, making microbes that naturally harbour these better hosts. A primary example is the metal-dependent formate dehydrogenase, which can support highly efficient utilization of formate as cellular energy source but is difficult to introduce into a new host (Maia et al., 2017).

In the next few years, we will likely witness the completion of synthetic $\mathrm{C} 1$ metabolism in model microbes. While we cannot predict which pathway will prove to be the most beneficial in the long term, it is clear that efforts such as those described in this review are bringing efficient bioproduction from $\mathrm{C}$ feedstocks closer.

\section{Acknowledgements}

The authors thank Charles Cotton for his feedback and critical reading of this manuscript. This work was funded by the MaxPlanck Society and European Commission Horizon 2020 grant eForFuel. Nico Claassens is supported by The Netherlands Organisation for Scientific Research (NWO) through a Rubicon Grant (Project 019.163LW.035). Hai He is funded by the China Scholarship Council.

\section{References}

Antonovsky, N., Gleizer, S., Noor, E., Zohar, Y., Herz, E., Barenholz, U., Zelcbuch, L., Amram, S., Wides, A., Tepper, N., et al. (2016). Sugar synthesis from $\mathrm{CO}_{2}$ in Escherichia coli. Cell 166, 115-125. https://doi. org/10.1016/j.cell.2016.05.064

Antonovsky, N., Gleizer, S., and Milo, R. (2017). Engineering carbon fixation in E. coli: from heterologous $\mathrm{RuBisCO}$ expression to the Calvin-Benson-Bassham cycle. Curr. Opin. Biotechnol. 47, 83-91.

Bar-Even, A. (2016). Formate assimilation: The metabolic architecture of natural and synthetic pathways. Biochemistry 55, 3851-3863. https://doi.org/10.1021/ acs.biochem.6b00495

Bar-Even, A., Noor, E., Flamholz, A., and Milo, R. (2013). Design and analysis of metabolic pathways supporting formatotrophic growth for electricitydependent cultivation of microbes. Biochim. Biophys.
Acta 1827, 1039-1047. https://doi.org/10.1016/j. bbabio.2012.10.013

Barenholz, U., Davidi, D., Reznik, E., Bar-On, Y., Antonovsky, N., Noor, E., and Milo, R. (2017). Design principles of autocatalytic cycles constrain enzyme kinetics and force low substrate saturation at flux branch points. Elife 6, e20667. https://doi.org/10.7554/eLife.20667

Bennett, R.K., Steinberg, L.M., Chen, W., and Papoutsakis, E.T. (2018a). Engineering the bioconversion of methane and methanol to fuels and chemicals in native and synthetic methylotrophs. Curr. Opin. Biotechnol. 50, 81-93.

Bennett, R.K., Gonzalez, J.E., Whitaker, W.B., Antoniewicz, M.R., and Papoutsakis, E.T. (2018b). Expression of heterologous non-oxidative pentose phosphate pathway from Bacillus methanolicus and phosphoglucose isomerase deletion improves methanol assimilation and metabolite production by a synthetic Escherichia coli methylotroph. Metab. Eng. 45, 75-85.

Bertsch, J., and Müller, V. (2015). Bioenergetic constraints for conversion of syngas to biofuels in acetogenic bacteria. Biotechnol. Biofuels 8, 210. https://doi. org/10.1186/s13068-015-0393-x

Bogorad, I.W., Chen, C.T., Theisen, M.K., Wu, T.Y., Schlenz, A.R., Lam, A.T., and Liao, J.C. (2014). Building carbon-carbon bonds using a biocatalytic methanol condensation cycle. Proc. Natl. Acad. Sci. U.S.A. 111, 15928-15933. https://doi.org/10.1073/ pnas. 1413470111

Bouzon, M., Perret, A., Loreau, O., Delmas, V., Perchat, N., Weissenbach, J., Taran, F., and Marlière, P. (2017). A Synthetic Alternative to Canonical One-Carbon Metabolism. ACS Synth. Biol. 6, 1520-1533. https:// doi.org/10.1021/acssynbio.7b00029

Burgard, A.P., Pharkya, P., and Maranas, C.D. (2003). Optknock: a bilevel programming framework for identifying gene knockout strategies for microbial strain optimization. Biotechnol. Bioeng. 84, 647-657. https:// doi.org/10.1002/bit.10803

Chen, C.T., Chen, F.Y., Bogorad, I.W., Wu, T.Y., Zhang, R., Lee, A.S., and Liao, J.C. (2018). Synthetic methanol auxotrophy of Escherichia coli for methanol-dependent growth and production. Metab. Eng. 49, 257-266.

Chistoserdova, L., Kalyuzhnaya, M.G., and Lidstrom, M.E. (2009). The expanding world of methylotrophic metabolism.Annu. Rev.Microbiol.63,477-499.https:// doi.org/10.1146/annurev.micro.091208.073600

Claassens, N.J., Sánchez-Andrea, I., Sousa, D.Z., and BarEven, A. (2018). Towards sustainable feedstocks: A guide to electron donors for microbial carbon fixation. Curr. Opin. Biotechnol. 50, 195-205.

Clomburg, J.M., Crumbley, A.M., and Gonzalez, R. (2017). Industrial biomanufacturing: The future of chemical production. Science 355, aag0804.

Dai, Z., Gu, H., Zhang, S., Xin, F., Zhang, W., Dong, W., Ma, J., Jia, H., and Jiang, M. (2017). Metabolic construction strategies for direct methanol utilization in Saccharomyces cerevisiae. Bioresour. Technol. 245, 1407-1412. https:// doi.org/10.1016/j.biortech.2017.05.100

Döring, V., Darii, E., Yishai, O., Bar-Even, A., and Bouzon, M. (2018). Implementation of a reductive route of onecarbon assimilation in Escherichia coli through directed 
evolution. ACS Synth. Biol. 7, 2029-2036. https://doi. org/10.1021/acssynbio.8b00167

Dürre, P., and Eikmanns, B.J. (2015). C1-carbon sources for chemical and fuel production by microbial gas fermentation. Curr. Opin. Biotechnol. 35, 63-72. https://doi.org/10.1016/j.copbio.2015.03.008

Erb, T.J., Jones, P.R., and Bar-Even, A. (2017). Synthetic metabolism: metabolic engineering meets enzyme design. Curr. Opin. Chem. Biol. 37, 56-62.

Fei, Q. Guarnieri, M.T., Tao, L., Laurens, L.M., Dowe, N., and Pienkos, P.T. (2014). Bioconversion of natural gas to liquid fuel: opportunities and challenges. Biotechnol. Adv. 32, 596-614. https://doi.org/10.1016/j. biotechadv.2014.03.011

Figueroa, I.A., Barnum, T.P., Somasekhar, P.Y., Carlström, C.I., Engelbrektson, A.L., and Coates, J.D. (2018). Metagenomics-guided analysis of microbial chemolithoautotrophic phosphite oxidation yields evidence of a seventh natural $\mathrm{CO}_{2}$ fixation pathway. Proc. Natl. Acad. Sci. U.S.A. 115, E92-E101. https:// doi.org/10.1073/pnas.1715549114

Gonzalez, J.E., Bennett, R.K., Papoutsakis, E.T., and Antoniewicz, M.R. (2018). Methanol assimilation in Escherichia coli is improved by co-utilization of threonine and deletion of leucine-responsive regulatory protein. Metab. Eng. 45, 67-74.

He, H., Edlich-Muth, C., Lindner, S.N., and Bar-Even, A. (2018). Ribulose monophosphate shunt provides nearly all biomass and energy required for growth of $E$. coli. ACS Synth. Biol. 7, 1601-1611. https://doi.org/10.1021/ acssynbio.8b00093

Henstra, A.M., Sipma, J., Rinzema, A., and Stams, A.J. (2007). Microbiology of synthesis gas fermentation for biofuel production. Curr. Opin. Biotechnol. 18, 200-206.

Herz, E., Antonovsky, N., Bar-On, Y., Davidi, D., Gleizer, S., Prywes, N., Noda-Garcia, L., Lyn Frisch, K., Zohar, Y., Wernick, D.G., et al. (2017). The genetic basis for the adaptation of $\mathrm{E}$. coli to sugar synthesis from $\mathrm{CO}_{2}$. Nat. Commun. 8, 1705. https://doi.org/10.1038/s41467017-01835-3

Jouny, M., Luc, W.W., and Jiao, F. (2018). A general technoeconomic analysis of $\mathrm{CO}_{2}$ electrolysis systems. Ind. Eng. Chem. Res. 57, 2165-2177. https://doi.org/10.1021/ acs.iecr.7b03514

Kato, N., Yurimoto, H., and Thauer, R.K. (2006). The physiological role of the ribulose monophosphate pathway in bacteria and archaea. Biosci. Biotechnol. Biochem. 70, 10-21.

Kerby, R., and Zeikus, J.G. (1987). Anaerobic catabolism of formate to acetate and $\mathrm{CO}_{2}$ by Butyribacterium methylotrophicum. J. Bacteriol. 169, 2063-2068.

Kopljar, D., Wagner, N., and Klemm, E. (2016). Transferring electrochemical $\mathrm{CO}_{2}$ reduction from semi-batch into continuous operation mode using gas diffusion electrodes. Chem. Eng. Technol. 39, 2042-2050. https://doi.org/10.1002/ceat.201600198

Kumar, B., Llorente, M., Froehlich, J., Dang, T., Sathrum, A., and Kubiak, C.P. (2012). Photochemical and photoelectrochemical reduction of CO2. Annu. Rev. Phys. Chem. 63, 541-569. https://doi.org/10.1146/ annurev-physchem-032511-143759
Leßmeier, L., Pfeifenschneider, J., Carnicer, M., Heux, S., Portais, J.C., and Wendisch, V.F. (2015). Production of carbon-13-labeled cadaverine by engineered Corynebacterium glutamicum using carbon-13-labeled methanol as co-substrate. Appl. Microbiol. Biotechnol. 99, 10163-10176. https://doi.org/10.1007/s00253015-6906-5

Maia, L.B., Moura, I., and Moura, J.J.G. (2017). Molybdenum and tungsten-containing formate dehydrogenases: Aiming to inspire a catalyst for carbon dioxide utilization. Inorganica Chim. Acta 455, 350363. https://doi.org/10.1016/j.ica.2016.07.010

Marlière, P., Patrouix, J., Döring, V., Herdewijn, P., Tricot, S., Cruveiller, S., Bouzon, M., and Mutzel, R. (2011). Chemical evolution of a bacterium's genome. Angew. Chem. Int. Ed. Engl. 50, 7109-7114. https://doi. org/10.1002/anie.201100535

Marmiesse, L., Peyraud, R., and Cottret, L. (2015). FlexFlux: combining metabolic flux and regulatory network analyses. BMC Syst. Biol. 9, 93. https://doi. org/10.1186/s12918-015-0238-z

Martín, A.J., Larrazábal, G.O., and Pérez-Ramírez, J. (2015). Towards sustainable fuels and chemicals through the electrochemical reduction of $\mathrm{CO} 2$ : lessons from water electrolysis. Green Chem. 17, 5114-5130. https://doi. org/10.1039/C5GC01893E

Meyer, F., Keller, P., Hartl, J., Gröninger, O.G., Kiefer, P., and Vorholt, J.A. (2018). Methanol-essential growth of Escherichia coli. Nat. Commun. 9, 1508. https://doi. org/10.1038/s41467-018-03937-y

Mohd Azhar, S.H., Abdulla, R., Jambo, S.A., Marbawi, H., Gansau, J.A., Mohd Faik, A.A., and Rodrigues, K.F. (2017). Yeasts in sustainable bioethanol production: A review. Biochem. Biophys. Rep. 10, 52-61. https://doi. org/10.1016/j.bbrep.2017.03.003

Müller, J.E.N., Meyer, F., Litsanov, B., Kiefer, P., Potthoff, E., Heux, S., Quax, W.J., Wendisch, V.F., Brautaset, T., Portais, J.C., et al. (2015). Engineering Escherichia coli for methanol conversion. Metab. Eng. 28, 190-201.

Neidhardt, F.C., Ingraham, J.L., and Schaechter, M. (1990). Building blocks needed to produce $1 \mathrm{~g}$ of $E$. coli protoplasm. In Physiology of the Bacterial Cell: A Molecular Approach, Neidhardt, F.C., Ingraham, J.L., and Schaechter, M., eds. (Sinaver Associates, Sunderland, MA), pp. 134-143.

Olah, G.A. (2013). Towards oil independence through renewable methanol chemistry. Angew. Chem. Int. Ed. Engl. 52, 104-107. https://doi.org/10.1002/ anie.201204995

Olah, G.A., Goeppert, A., and Prakash, G.K.S. (2009). Beyond Oil and Gas: The Methanol Economy, Second Edition. (Wiley VCH, Weinheim), pp. 1-334 https:// doi.org/10.1002/9783527627806.

Overkamp, K.M., Kötter, P., van der Hoek, R., Schoondermark-Stolk, S., Luttik, M.A., van Dijken, J.P., and Pronk, J.T. (2002). Functional analysis of structural genes for $\mathrm{NAD}(+)$-dependent formate dehydrogenase in Saccharomyces cerevisiae. Yeast 19, 509-520.

Pfeifenschneider, J., Brautaset, T., and Wendisch, V.F. (2017). Methanol as carbon substrate in the bio-economy: Metabolic engineering of aerobic methylotrophic bacteria for production of value-added 
chemicals. Biofuels Bioprod. Biorefining 11, 719-731. https://doi.org/10.1002/bbb.

Portnoy, V.A., Bezdan, D., and Zengler, K. (2011). Adaptive laboratory evolution - harnessing the power of biology for metabolic engineering. Curr. Opin. Biotechnol. 22, 590-594. https://doi.org/10.1016/j. copbio.2011.03.007

Price, J.V., Chen, L., Whitaker, W.B., Papoutsakis, E., and Chen, W. (2016). Scaffoldless engineered enzyme assembly for enhanced methanol utilization. Proc. Natl. Acad. Sci. U.S.A. 113, 12691-12696. https://doi. org/10.1073/pnas.1601797113

Quayle, J.R., and Ferenci, T. (1978). Evolutionary aspects of autotrophy. Microbiol. Rev. 42, 251-273.

Schrader, J., Schilling, M., Holtmann, D., Sell, D., Filho, M.V., Marx, A., and Vorholt, J.A. (2009). Methanolbased industrial biotechnology: current status and future perspectives of methylotrophic bacteria. Trends Biotechnol. 27, 107-115. https://doi.org/10.1016/j. tibtech.2008.10.009

Schwander, T., Schada von Borzyskowski, L., Burgener, S., Cortina, N.S., and Erb, T.J. (2016). A synthetic pathway for the fixation of carbon dioxide in vitro. Science 354, 900-904.

Shen, Y., Jarboe, L., Brown, R., and Wen, Z. (2015). A thermochemical-biochemical hybrid processing of lignocellulosic biomass for producing fuels and chemicals. Biotechnol. Adv. 33, 1799-1813. https://doi. org/10.1016/j.biotechadv.2015.10.006

Siegel, J.B., Smith, A.L., Poust, S., Wargacki, A.J., Bar-Even, A., Louw, C., Shen, B.W., Eiben, C.B., Tran, H.M., Noor, E., et al. (2015). Computational protein design enables a novel one-carbon assimilation pathway. Proc. Natl. Acad. Sci. U.S.A. 112, 3704-3709. https://doi.org/10.1073/ pnas. 1500545112

Tashiro, Y., Hirano, S., Matson, M.M., Atsumi, S., and Kondo, A. (2018). Electrical-biological hybrid system for $\mathrm{CO} 2$ reduction. Metab. Eng. 47, 211-218.

Tuyishime, P., Wang, Y., Fan, L., Zhang, Q. Li, Q. Zheng, P., Sun, J., and Ma, Y. (2018). Engineering Corynebacterium glutamicum for methanol-dependent growth and glutamate production. Metab. Eng. 49, 220-231.

Wang, W.H., Himeda, Y., Muckerman, J.T., Manbeck, G.F., and Fujita, E. (2015). $\mathrm{CO}_{2}$ hydrogenation to formate and methanol as an alternative to photo- and electrochemical $\mathrm{CO}_{2}$ reduction. Chem. Rev. 115, 12936-12973. https:// doi.org/10.1021/acs.chemrev.5b00197

Wang, X., Wang, Y., Liu, J., Li, Q. Zhang, Z., Zheng, P., Lu, F., and Sun, J. (2017). Biological conversion of methanol by evolved Escherichia coli carrying a linear methanol assimilation pathway. Bioresour. Bioprocess. 4, 4-9. https://doi.org/10.1186/s40643-017-0172-6.

Wendisch, V.F., Bott, M., and Eikmanns, B.J. (2006). Metabolic engineering of Escherichia coli and Corynebacterium glutamicum for biotechnological production of organic acids and amino acids. Curr. Opin. Microbiol. 9, 268-274.

Wenk, S., Yishai, O., Lindner, S.N., and Bar-Even, A. (2018). An engineering approach for rewiring microbial metabolism. Meth. Enzymol. 608, 329-367.

Whitaker, W.B., Sandoval, N.R., Bennett, R.K., Fast, A.G., and Papoutsakis, E.T. (2015). Synthetic methylotrophy: engineering the production of biofuels and chemicals based on the biology of aerobic methanol utilization. Curr. Opin. Biotechnol. 33, 165-175. https://doi. org/10.1016/j.copbio.2015.01.007

Whitaker, W.B., Jones, J.A., Bennett, R.K., Gonzalez, J.E., Vernacchio, V.R., Collins, S.M., Palmer, M.A., Schmidt, S., Antoniewicz, M.R., Koffas, M.A., et al. (2017). Engineering the biological conversion of methanol to specialty chemicals in Escherichia coli. Metab. Eng. 39, 49-59.

Witthoff, S., Schmitz, K., Niedenführ, S., Nöh, K., Noack, S., Bott, M., and Marienhagen, J. (2015). Metabolic engineering of Corynebacterium glutamicum for methanol metabolism. Appl. Environ. Microbiol. 81, 2215-2225. https://doi.org/10.1128/AEM.03110-14

Woolston, B.M., King, J.R., Reiter, M., Van Hove, B., and Stephanopoulos, G. (2018a). Improving formaldehyde consumption drives methanol assimilation in engineered E. coli. Nat. Commun. 9, 2387. https://doi. org/10.1038/s41467-018-04795-4

Woolston, B.M., Roth, T., Kohale, I., Liu, D.R., and Stephanopoulos, G. (2018b). Development of a formaldehyde biosensor with application to synthetic methylotrophy. Biotechnol. Bioeng. 115, 206-215. https://doi.org/10.1002/bit.26455

Yang, H., Kaczur, J.J., Sajjad, S.D., and Masel, R.I. (2017). $\mathrm{CO} 2$ Conversion to formic acid in a three compartment cell with Sustainion ${ }^{\mathrm{TM}}$ membranes. ECS Trans. 77, 1425-1431. https://doi.org/10.1149/07711.1425ecst.

Yishai, O., Goldbach, L., Tenenboim, H., Lindner, S.N., and Bar-Even, A. (2017). Engineered assimilation of exogenous and endogenous formate in Escherichia coli. ACS Synth. Biol. 6, 1722-1731. https://doi. org/10.1021/acssynbio.7b00086

Yishai, O., Bouzon, M., Döring, V., and Bar-Even, A. (2018). In vivo assimilation of one-carbon via a synthetic reductive glycine pathway in Escherichia coli. ACS Synth. Biol. 7, 2023-2028. https://doi.org/10.1021/ acssynbio.8b00131

Zelcbuch, L., Antonovsky, N., Bar-Even, A., Levin-Karp, A., Barenholz, U., Dayagi, M., Liebermeister, W., Flamholz, A., Noor, E., Amram, S., et al. (2013). Spanning highdimensional expression space using ribosome-binding site combinatorics. Nucleic Acids Res. 41, e98. https:// doi.org/10.1093/nar/gkt151

Zelcbuch, L., Razo-Mejia, M., Herz, E., Yahav, S., Antonovsky, N., Kroytoro, H., Milo, R., and Bar-Even, A. (2015). An in vivo metabolic approach for deciphering the product specificity of glycerate kinase proves that both E. coli's glycerate kinases generate 2-phosphoglycerate. PLOS ONE 10, e0122957. https://doi.org/10.1371/journal. pone.0122957

Zhang, W., Zhang, T., Wu, S., Wu, M., Xin, F., Dong, W., Ma, J., Zhang, M., and Jiang, M. (2017). Guidance for engineering of synthetic methylotrophy based on methanol metabolism in methylotrophy. RSC Adv. 7, 4083-4091. https://doi.org/10.1039/C6RA27038G

Zhou, X., Liu, R., Sun, K., Chen, Y., Verlage, E., Francis, S.A., Lewis, N.S., and Xiang, C. (2016). Solar-driven reduction of $1 \mathrm{~atm}$ of $\mathrm{CO}_{2}$ to formate at $10 \%$ energy-conversion efficiency by use of a TiO2-protected III-V tandem photoanode in conjunction with a bipolar membrane and a Pd/C cathode. ACS Energy Lett. 1, 764-770. https://doi.org/10.1021/acsenergylett.6b00317 